\title{
A Lightweight Framework for Intelligent Fault Diagnosis Acceleration in Complex Industrial Systems
}

This paper was downloaded from TechRxiv (https://www.techrxiv.org).

LICENSE

CC BY 4.0

SUBMISSION DATE / POSTED DATE

$15-11-2021 / 17-11-2021$

\section{CITATION}

Shan, Nanliang (2021): A Lightweight Framework for Intelligent Fault Diagnosis Acceleration in Complex Industrial Systems. TechRxiv. Preprint. https://doi.org/10.36227/techrxiv.17009621.v1

DOI

10.36227/techrxiv.17009621.v1 


\title{
A Lightweight Framework for Intelligent Fault Diagnosis Acceleration in Complex Industrial Systems
}

\author{
Nanliang Shan, Student Member, IEEE, Xinghua Xu, Shaohua Qiu and Xianqiang Bao, \\ Member, IEEE
}

\begin{abstract}
With the acquisition of massive condition monitoring data, how to realize real-time and efficient intelligent fault diagnosis is the focus of current research. Inspired by the ideas of compressed sensing (CS) and deep extreme learning machines (DELM), a data-driven lightweight framework is proposed to accelerate intelligent fault diagnosis. The integrated framework contains two modules: data sampling and fault diagnosis. Data sampling module projects the intensive original monitoring data into lightweight compressed sampling data non-linearly, which can effectively reduce the pressure of transmission, storage and calculation. Fault diagnosis module digs deeply into the inner connection between the compressed sampled signal and the fault types to realize accurate fault diagnosis. This work has three meaningful points. First, we believe that the bearing vibration signal is not strictly sparse in the transform domain. Second, we verified that the sparse signal after compressed sampling can be directly used for fault diagnosis without being reconstructed. Third, adding a kernel function to the DELM can perfectly map the low-dimensional inseparable features after compressed sampling to the high-dimensional space non-linearly to make it linearly separable and thus improve the classification accuracy.
\end{abstract}

Index Terms-Compressed sensing, deep kernel extreme learning machine, intelligent fault diagnosis, lightweight computing.

\section{INTRODUCTION}

$\mathrm{W}$ ITH the development of modern industrial systems and the human pursuit of extreme efficiency, complex industrial systems are gradually automated, sophisticated and integrated [1]. This also leads to the mechanical fault of some key components, which will cause the paralysis of the entire system. To accurately grasp the internal status information of key components, high-speed real-time online sampling methods are currently used to obtain time-series monitoring signals for health status assessment. Due to the many monitoring points, high sampling rate, and long monitoring time, the health management system has acquired massive

Manuscript received Month $x x, 2 x x x$; revised Month $x x, x x x x$; accepted Month $x, x x x x$. This work was supported in part by the National Natural Science Foundation of China under Grant 62102436 (Corresponding author: Xianqiang Bao, baoxianqiang@nudt.edu.cn ).

N.L. Shan, X.H. Xu, S.H. Qu and X.Q. Bao are with the National Key Laboratory of Science and Technology on Vessel Integrated Power System, Naval University of Engineering, Wuhan, 430033, China (e-mail: nanliang@stu.xmu.edu.cn). status data. On one hand, it has prompted the fault diagnosis of complex industrial systems to move into the "data-driven" era [2], on the other hand, it has also caused great difficulty in real-time and accurate fault diagnosis of key components [3].

At present, the health monitoring of complex industrial systems mainly adopts the "data-driven" intelligent fault diagnosis method [4-6], including three steps of data sampling, feature extraction, and fault classification. Data sampling refers to monitoring the health status of the target device for a long time through a large number of sensors. The current data sampling methods follow the Nyquist sampling theorem, that is, the original signal is uniformly sampled at twice the signal frequency at equal intervals [7]. Feature extraction refers to obtaining data features from a signal through signal processing methods, mainly in the time domain, frequency domain, and transform domain methods. Transform domain analysis methods have been widely used in processing non-stationary signals such as mechanical vibration signals, which include principal component analysis [8], discrete cosine transform [9], and so on. Fault classification refers to predicting the health status or fault category of unknown signals by learning data features. The research on fault classification methods is relatively mature, which include the classic fault classification algorithms include artificial neural network (ANN) [10], support vector machine (SVM) [11], K-mean [12], fuzzy inference system [13], etc.

Most research on intelligent fault diagnosis have achieved valuable results. However, three obvious shortcomings are worthy of further research.

1) The sampled signal obtained based on the Nyquist theorem contains a lot of redundant information, which will greatly increase the pressure for subsequent data transmission, storage, and calculation.

2) Feature extracted from massive sampled signals obtained by Nyquist sampling has problems in subsequent processing, such as high computational complexity, time consuming, and incomplete reflection of fault features.

3) The structure of the traditional fault classification model is too simple to effectively obtain the complex nonlinear relationships hidden in the data. And the structure of the emerging DNN model is too complex to recognize sensitive features in real time under a resource-constrained environment.

It is very important to change the data processing mode of the existing real-time monitoring system fundamentally, so a new theory on data sampling and compression came into being 
- Compressed Sensing (CS) [14]. CS adopts an approximately optimal sampling scheme, theoretically obtains all the information contained in the original signal and effectively realizes the dimensionality reduction of the signal. It frees data sampling from the Nyquist theory and can achieve signal compression sampling through nonlinear projection in the transform domain, thereby reducing the mass of redundant data sampling into a small amount of useful information sampling. Therefore, the storage and calculation costs required for feature extraction are greatly reduced. Integrating data sampling and compression processing, the new information sensing method provides a brand-new idea for data sampling and processing in real-time monitoring systems.

Some studies have introduced the concept of CS [15-17], and the general process of which includes compressed sampling, data transmission, and compressed signal reconstruction. Then, the reconstructed signal will be input to the feature extraction and fault classification model. Although these methods reduce the requirements for data storage and data transmission, the subsequent operations are still based on the reconstructed time-domain signals, hence the model training time and fault diagnosis accuracy still have much room for improvement. So we thought about whether the compressed sampling signal can be directly used for fault diagnosis? This question is not difficult to answer. Since the compressed sampling signals contain all the information of the fault characteristics, efficient fault diagnosis can be realized as long as a suitable fault diagnosis model is constructed.

Deep extreme learning machine (DELM) [18] is a multi-layer neural network that uses extreme learning machine-autoencoder (ELM-AE) as the basic unit of unsupervised learning, which performs greedy layer-by-layer unsupervised training on input data. It is widely used because it is easier than other deep neural networks in terms of model training, generalization ability, and weight and bias update of hidden layers. Compared with the ELM-AE that hsa a single hidden layer, the DELM can extract more complex fault features in the low-dimensional observation signal. Therefore, the DELM is an ideal fault classification model that integrates the accuracy of DNN and the speed of traditional machine learning.

Combining the advantages of CS and DELM, this paper proposes an intelligent diagnosis framework to realize the functional integration of signal compressed sampling, feature adaptive extraction and fault classification. The kernel function is introduced to map the low-dimensional non-linear inseparable data feature to the high-dimensional space to make it linearly separable, so as to achieve more accurate fault classification. Finally, the PSO algorithm [19] is used to optimize the nodes of each hidden layer, the regularization coefficient, the penalty coefficient and the core parameters of the KELM, so as to achieve the best fault classification results. The main contributions of this paper are summarized as follows:

1) A data-driven lightweight framework for accelerating intelligent fault diagnosis is proposed. The framework reduces the intensive original sampled data into a small amount of compressed sampled data, which effectively relieves the calculation and storage pressure in the fault diagnosis process. At the same time, the fault diagnosis module of the framework integrates DELM, kernel functions, and PSO to deeply explore the internal connections between compressed sampling signals and fault types to achieve accurate fault diagnosis and classification.

2) A new adaptive feature extraction method for bearing vibration signals is proposed. Our research find that the bearing vibration signal is not strictly sparse in the transform domain, so it is not suitable to use CS on the original time-domain signal directly. Instead, a sparse basis should be added before the compressed sampling, aiming to transform the time-domain signal into a certain transformation domain. And then an observation matrix that is incoherent to the sparse basis should be used to project the transformed high-dimensional signal onto a low-dimensional space. This can be seen as a feature representation.

3) An improved DKELM fault diagnosis model is constructed to mine the complex mapping between measurement data and fault types. The effects of key parameter selection of the model on fault diagnosis were also deeply studied. This model is suitable for sparse signals after compressed sampling and can use only a small number of sampled signals to diagnose fault types. The comparison with the existing intelligent fault diagnosis methods reflects the superiority of proposed model. It also provides a new idea for intelligent fault diagnosis.

The remainder of this paper is organized as follows. Section II briefly introduces the theoretical basis of CS and DKELM. Section III introduces the CS-DKELM intelligent fault diagnosis framework proposed in this paper. Section IV verifies the effectiveness of the framework. In addition, our method is compared with existing methods, and the effects of important factors are analyzed. Finally, Section V states the conclusion.

\section{THEORETICAL BACKGROUND}

\section{A. CS}

In view of the large scale of sampled signals in complex industrial systems, it is inconvenient to transmit, store and calculate. Donoho [14] proposed a Compressed Sensing (CS) theory based on signal sparsity, which broke the traditional lossless signal restoration theory based on signal bandwidth as a priori (Nyquist theory). CS utilizes the sparsity of the signal transform domain. It uses a measurement matrix that is incoherent to the transform domain to project original signal, then obtains a low-dimensional compressed sampling signal. The original signal can be restored with high fidelity through the combined optimization method. As a new sampling theory, by combining the process of compression and sampling, the sampling amount of the original signal is greatly reduced on the premise of ensuring signal integrity. The compressed sampling signal can be expressed as follows:

$$
\mathrm{y}=\Phi \mathrm{x}=\Phi \Psi_{\mathrm{s}}
$$


where $\Phi \in R^{M \times N}(M \ll N)$ is the measurement matrix. $x \in R^{N}$ is the original signal. $\Psi$ is the dictionary matrix or sparse basis. $\mathrm{s}$ is the sparse factor, which contains a few nonzero values.

In the process of $\mathrm{CS}$, the Compression Rate (CR) reflects the degree of compression of the original signal, which is defined as follows:

$$
\mathrm{CR}=\frac{\mathrm{N}-\mathrm{M}}{\mathrm{N}} \times 100 \%
$$

where $\mathrm{N}$ is the dimension of the original signal. $\mathrm{M}$ is the dimension of the compressed signal. By adjusting the $\mathrm{CR}$, different degrees of compression can be achieved. The larger the $\mathrm{CR}$ is, the less compressed sampling data can be obtained.

There are two prerequisites for the realization of CS. One is that the original signal must meet the sparseness in a certain transform domain [14], and the other is that the measurement matrix must meet the restricted isometric property (RIP) [20]. The compressed sampling data that meets these two conditions can be accurately reconstructed. Candes E [21] proved that the Random Gaussian Matrix can satisfy RIP with high probability and has good universality, so it is widely used in CS. This feature makes it possible to generate a Random Gaussian Matrix of appropriate size as a measurement matrix to compress and sample the original signal when the signal is sparse in the unknown space.

\section{B. ELM}

Extreme Learning Machine (ELM) [22] is proposed for the training of a single hidden layer feedforward neural network. It is very different from the traditional BP network. It does not need to solve iteratively but adopts a batch-processing "one-step" training method. Randomly select the weights and biases of hidden nodes and complete the training by the inverse operation of the output weights. It greatly improves the generalization ability and training speed of the network, also reduces the amount of calculation and search space.

Giving $N$ training samples

$$
Z=\left\{x_{i}, t_{i}\right\}_{i=1}^{N}, x_{i} \in R^{n}, t_{i} \in R^{m}
$$

Then the ELM model with $L$ nodes can be expressed as

$$
\sum_{j=1}^{L} \beta_{j} g\left(\omega_{j} x_{i}+b_{j}\right)=t_{i}
$$

where $L$ is the hidden layer nodes. $\omega_{j}=\left[\omega_{j 1}, \omega_{j 2}, \cdots, \omega_{j n}\right]^{T}$ is the weight vector connecting the $j t h$ hidden layer node and the input nodes. $\beta_{j}=\left[\beta_{j 1}, \beta_{j 2}, \cdots, \beta_{j m}\right]^{T}$ is the weight vector connecting the $j$ th hidden layer node and the output nodes. $g()$ is the activation function. The above formula can be abbreviated as

$$
\boldsymbol{H} \boldsymbol{\beta}=\boldsymbol{T}
$$

where $\boldsymbol{H}$ is $N \times L$ hidden layer output matrix. $\boldsymbol{\beta}=$ $\left[\beta_{1}, \beta_{2}, \cdots, \beta_{L}\right]_{L \times m}^{T}$ is the output weight matrix. $\boldsymbol{T}=$ $\left[t_{1}, t_{2}, \cdots, t_{n}\right]_{N \times m}^{T}$ is the label corresponding to the input sample. Thus we have

$$
\boldsymbol{\beta}=\boldsymbol{H}^{\dagger} \boldsymbol{T}
$$

Here $\boldsymbol{H}^{\dagger}=\boldsymbol{H}^{T}\left(\boldsymbol{H} \boldsymbol{H}^{T}\right)^{-1}$ represents the Moore-Penrose Generalized Inverse of the hidden layer output matrix $\boldsymbol{H}$. To improve the generalization ability of the algorithm, the regularization parameter $C$ is introduced in Eq. (6), and the output weight matrix can be solved by the following

$$
\boldsymbol{\beta}=\boldsymbol{H}^{T}\left(\frac{\boldsymbol{I}}{\boldsymbol{C}}+\boldsymbol{H} \boldsymbol{H}^{T}\right)^{-1} \boldsymbol{T}
$$

Then the output of the ELM can be expressed as

$$
f(x)=h(x) \boldsymbol{H}^{T}\left(\frac{\boldsymbol{I}}{\boldsymbol{C}}+\boldsymbol{H} \boldsymbol{H}^{T}\right)^{-1} \boldsymbol{T}
$$

\section{DKELM}

The kernel function [23] has been applied to many problems due to its strong nonlinear mapping ability, and its most representative application is the Support Vector Machine (SVM) [24]. The kernel function can overcome the curse of dimensionality, and the samples that are linearly inseparable in the original space can be non-linearly mapped to a higher-dimensional space to make them linearly separable, thus improving the classification accuracy. Based on the superior performance of the kernel function, Huang [25] introduced the kernel function into the ELM and proposed the KELM algorithm to further enhance the generalization ability and stability. In this paper, the kernel function and DELM are combined to construct a Deep Kernel Extreme Learning machine (DKELM). The structure is shown in Fig. 1.

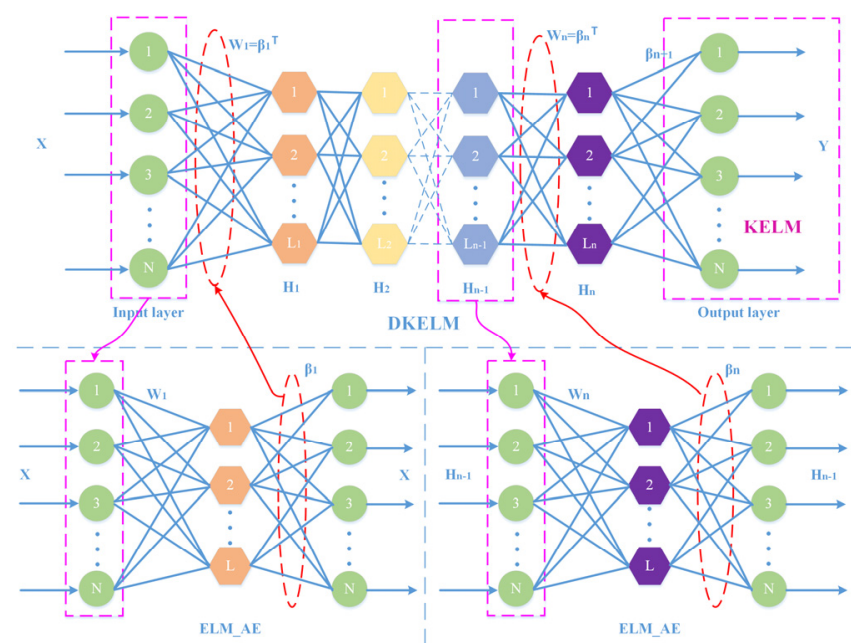

Fig. 1. Structure diagram of DKELM

The original input data of DKELM is abstracted by $k$ hidden layers to the input feature $X^{k}$, and then the kernel function maps the feature $X^{k}$. Then there is no need to give its specific form for the output matrix of KELM, just use the inner product principle of the kernel function to calculate the expression form of $H H^{T}$ and $h(x) H^{T} . H H^{T}$ can be expressed by the kernel function $K(x, y)$ as follows

$$
\left\{\begin{array}{l}
\Omega_{E L M}=H H^{T} \\
\Omega_{i, j}=h\left(x_{i}\right) \cdot h\left(x_{j}\right)=K\left(x_{i}, x_{j}\right)
\end{array}\right.
$$

Since $h(x) H^{T}$ can be expressed as

$$
h(x) H^{T}=\left[\begin{array}{c}
K\left(x, x_{1}\right) \\
\cdots \\
K\left(x, x_{N}\right)
\end{array}\right]
$$

The output of DKELM should be

$$
f(x)=\left[\begin{array}{c}
K\left(x, x_{1}\right) \\
\cdots \\
K\left(x, x_{N}\right)
\end{array}\right]\left(\frac{\boldsymbol{I}}{\boldsymbol{C}}+\Omega_{E L M}^{T}\right)^{-1} \boldsymbol{T}
$$

This is equivalent to using a kernel function to replace the mapping of the hidden nodes. The above-mentioned kernel 
function satisfies the Mercer theorem [26], so this paper adopts a widely used RBF kernel [27] form

$$
K\left(x, x_{i}\right)=\exp \left(-\frac{\left\|x-x_{i}\right\|^{2}}{\gamma}\right)
$$

where $\gamma$ is the nuclear parameter. The specific form of the feature mapping function $\mathrm{h}(\mathrm{x})$ of the hidden layer node in DKELM does not need to be specifically given, but only the specific form of the kernel function $K(x, x)$ can be used to find the value of the output function. Based on these abstract features rather than the original sample data, the kernel function calculation is used to replace the inner product operation in the high-dimensional space, then map the feature to the higher-dimensional space for accurate classification.

\section{PROPOSED CS-DKELM FRAMEWORK}

In view of the limitations of excessive data volume in the process of monitoring the health status of mechanical components, this paper proposes an integrated framework for data sampling and fault diagnosis based on CS and DKELM. The framework includes an original signal compressed sampling module based on CS, which achieves substantial compression under the premise of retaining fault information. It also contains a DKELM module based on ELM and kernel functions. Through multi-layer nonlinear learning of low-dimensional sampling values, it realizes the adaptive extraction of fault features and intelligent diagnosis of health status. The procedure of the proposed framework is shown in Fig. 2.

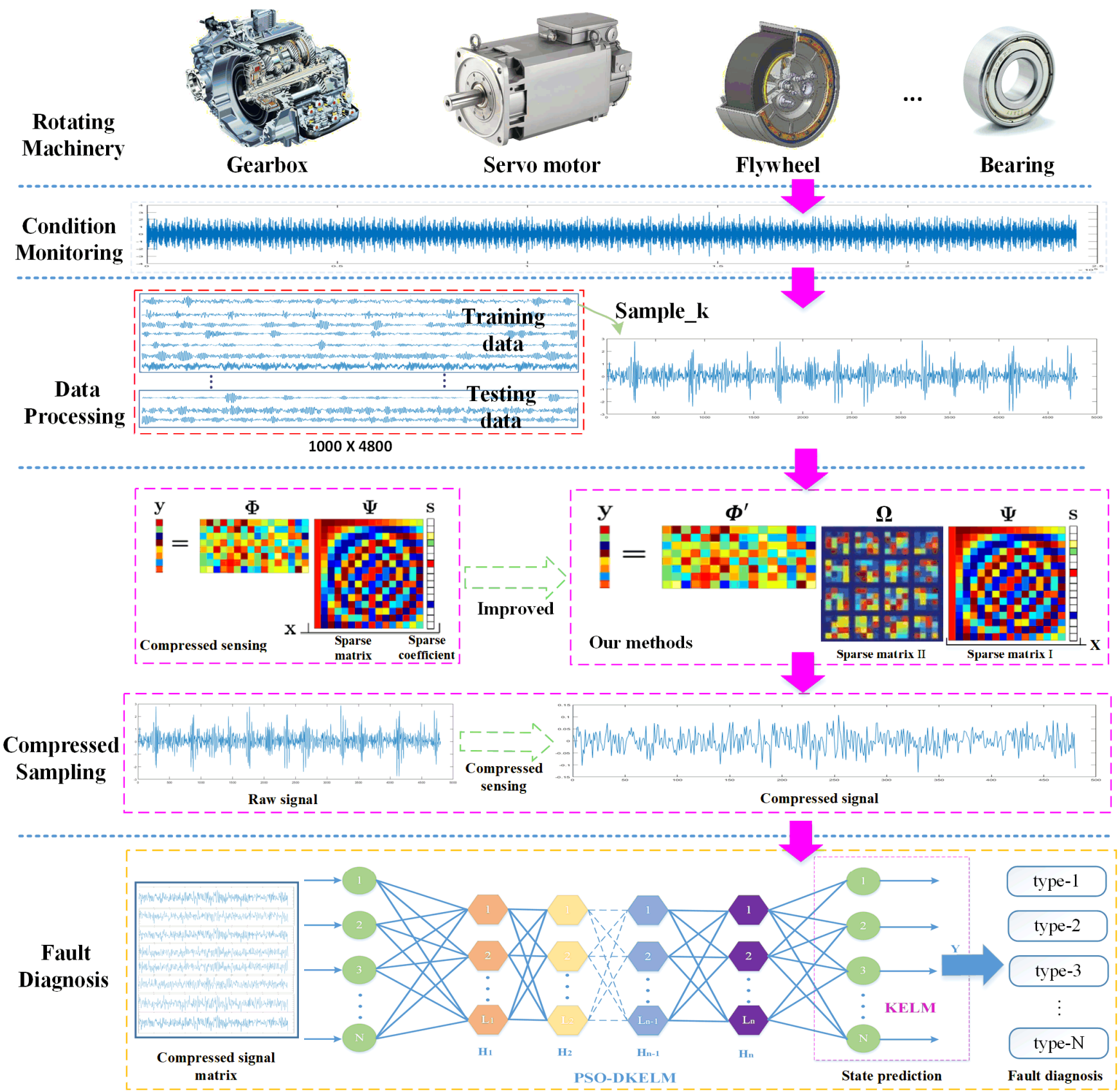

Fig. 2. The procedure of the proposed CS-DKELM framework 
1) The mechanical vibration signal is a high-frequency sampling signal. Calculating it directly will exceed the maximum length preset by the ordinary computer array, resulting in memory overflow. Therefore, the data must be preprocessed into data samples. This framework uses the sliding window to sample data and integrate the sampled time series data into a data set $D=\left\{d^{i}, l^{i}\right\}_{i=1}^{M}$, where $d^{i}$ is the sample data, $l^{i}$ is the corresponding label data, and $M$ is the total number of samples. The data sample set can be randomly selected and divided into a training set $D_{\text {train }}$ and a test set $D_{\text {test }}$.

2) Our research found that the bearing vibration signal is not strictly sparse in the transform domain. So, CS cannot be used directly on the original mechanical vibration signal. This framework adds a Fourier transform matrix before compressed sampling to transform the time-domain signal into the frequency-domain, and then uses an observation matrix (Gaussian random matrix) that is not incoherent to the sparse matrix (Discrete cosine transform matrix) to project the transformed frequency-domain signal onto a low-dimensional space to achieve compressed sampling. The obtained signal is a frequency-domain compressed sampling signal, which contains almost all the fault information of the original signal and can be directly used in the subsequent fault diagnosis process.

3) This framework trains the DKELM network with a layer-by-layer greedy method and saves the ELM-AE output weights obtained by the least square method. The input weights of each hidden layer are initialized with the output weights of ELM-AE, and layered unsupervised training is performed. DKELM does not require a reverse fine-tuning process, so it is characterized by fast speed. The idea of DKELM is to minimize the reconstruction error so that the output can be infinitely close to the original input. After training by each layer, it can learn more advanced features of the original data. Figure 1 describes the training process of the DKELM model. The input sample $X$ is used as the target output of the first ELM-AE $X_{1}=X$. Then the output weight $\beta_{1}$ of the first ELM-AE is used as the input weight $W_{1}$ of the first hidden layer. The output matrix $H_{1}$ of the first hidden layer is used as the input and target output $\left(H_{1}=X_{1}=X\right)$ of the next ELM-AE, and so on for training layer by layer. The last hidden layer is trained with KELM, $H_{i}$ is the output matrix of the last hidden layer, and Eq. 11 is used to solve the output weight of DKELM. Because of the application of the kernel function, data features that were originally low-dimensional linearly inseparable can be mapped to high-dimensional linearly separable space, making the classification more accurate. Lastly, the PSO algorithm is used to optimize the number of hidden layer nodes, the regularization coefficient, the penalty coefficient and kernel parameters of KELM.

\section{EXPERIMENTAL VALIDATION}

\section{A. Compressed Sampling}

The experimental data provided by the public bearing data set of Case Western Reserve University Bearing Data Center [28]. This data set is the drive end vibration data sampled by SKF6205-2RS deep groove ball bearing at a sampling frequency of $48 \mathrm{kHz}$ and a load of $0 \mathrm{HP}$. It includes 4 health conditions: Normal status $(\mathrm{N})$, Outer race fault $(\mathrm{O})$, Inner race fault (I), and Ball fault (B). Among them, the degree of fault is divided into 3 categories according to the damage diameter, which are $0.07,0.14$, and $0.21 \mathrm{~mm}$ respectively.

Because data of each type is too long to be directly compressed sampling, a sliding window is used to make data samples. The window length are 4800 sampling points, and the step size is random. 100 samples of each type of fault are collected. Mark the preprocessed data set as $D$, then the dimension of which is $1000 \times 4800$. Randomly select $70 \%$ of the data as training data, and the remaining $30 \%$ as test data. Then the preprocessed data set $D$ is compressed sampled and normalized. The measurement matrix is a Gaussian random matrix, and the compression rate $\mathrm{CR}=80 \%$. The compressed sampling data set $D^{\prime}$ is obtained, with a dimension of $1000 \times 960$. In subsequent experiments, the data set $D^{\prime}$ will be used as the input data for intelligent fault diagnosis. The details are shown in Table I.

TABLE I

BEARING DATA SET D/ D'

\begin{tabular}{cccccc}
\multicolumn{5}{c}{ BEARING DATA SET D/ D' } \\
\hline \hline $\begin{array}{c}\text { Data } \\
\text { set }\end{array}$ & Dimension & Number & $\begin{array}{c}\text { Fault } \\
\text { type }\end{array}$ & $\begin{array}{c}\text { Fault } \\
\text { diameter/mm }\end{array}$ & Label \\
\hline & & 100 & B & 0.07 & 1 \\
& 100 & B & 0.14 & 2 \\
& & 100 & B & 0.21 & 3 \\
& & 100 & I & 0.07 & 4 \\
${\boldsymbol{D} / \boldsymbol{D}^{\prime}}^{4} 4800 / 960$ & 100 & I & 0.14 & 5 \\
& & 100 & I & 0.21 & 6 \\
& & 100 & O & 0.07 & 7 \\
& & 100 & O & 0.14 & 8 \\
& & 100 & O & 0.21 & 9 \\
& & 100 & N & 0 & 10 \\
\hline \hline
\end{tabular}

\section{B. Recoverability of Compressed Sampling Signal}

Compared with traditional Nyquist sampling, CS can compress the original signal more thoroughly. Through random unequal interval sampling, the sampling frequency can be far less than the Nyquist requirement. This will greatly reduce the amount of sampling data, thereby reducing the data storage space and the amount of calculation. As the main feature information, the non-zero value of the transform domain will not decrease. Although many incoherent interference values will be generated, we can extract the non-zero value from the transform domain through the iteration of "Threshold detection - Calculating larger interference-Eliminating interferenceLowering the threshold". Thereby, the original signal can be restored with high fidelity. As shown in Fig. 3, the fault signal of the outer race is taken as an example for reconstruction (The amplitude of outer race changes greatly, so the reconstruction is 
the most difficult among all circumstances). From top to bottom are the comparison curves between the original signal and the reconstructed signal with compression ratios of $0.9,0.8$, and 0.5 . The orthogonal basis of compressed sampling is the DCT basis, and the measurement matrix is a Gaussian random matrix. The essence of signal reconstruction is to solve the minimum value of the L1 norm.

To evaluate the error of the reconstructed signal more accurately, this paper uses the RMSE as the error measurement index:

$$
R M S E=\sqrt{\frac{1}{N} \sum_{n=1}^{N}(x(n)-\hat{x}(n))^{2}}
$$

where $x(n)$ is the original signal, and $\hat{x}(n)$ is the reconstructed signal. Fig. 4 shows the RMSE curve of four fault conditions under different compression ratios.
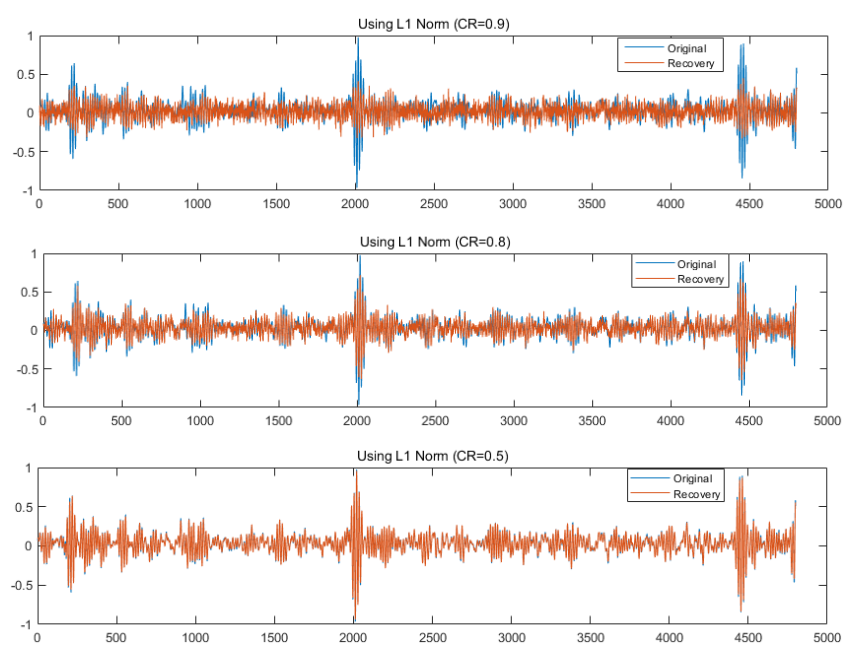

Fig. 3. Reconstructed waveform of outer race fault.

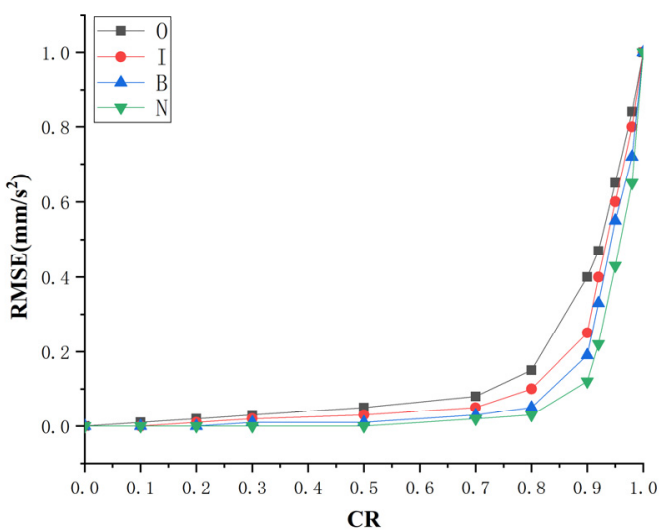

Fig. 4. The RMSE curve of the reconstructed signal.

When the compression ratio is 0.5 , the reconstructed signal can completely restore the waveform of the original signal's fault shock characteristics, and the RMSE at this time is mainly caused by the noise contained in the original signal. When the compression ratio is 0.8 , the reconstructed signal can still better reflect the mainshock characteristics, and the signal is slightly distorted. When the compression ratio is 0.9 , the reconstructed signal is severely distorted, and the fault shock characteristics cannot be extracted. Combined with the RMSE curve of the reconstructed signal, for the bearing vibration signal used in this paper, as long as the compression ratio is kept below 0.8 , the shock characteristic waveform in the original vibration signal can be accurately reconstructed.

\section{The Accuracy and Real-time of CS-DKELM}

The use of the CS-DKELM framework can greatly improve the accuracy and real-time capability of fault diagnosis. To verify the effectiveness of the intelligent diagnosis framework proposed in this paper, 300 test samples randomly selected in the early stage were input into the CS-DKELM framework to test the accuracy and real-time performance of fault diagnosis.

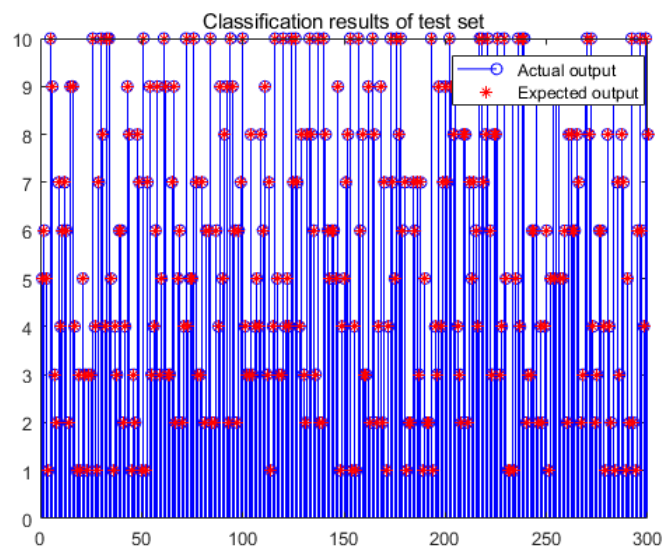

Fig. 5. Fault classification result of test set.

The classification result of the test set is shown in Fig. 5. The actual output result is completely consistent with the expected output result (the data label), and the fault classification accuracy rate can reach $100 \%$. To ensure the reliability of the experimental results, the average value of 20 experiments is adopted. A comparison with the method of directly identifying time-domain signals [29] is added, and the results are shown in Table II.

TABLE II

COMPARISON BETWEEN OUR METHOD AND THE TIME-DOMAIN METHOD

\begin{tabular}{ccccc}
\hline \hline Method & $\begin{array}{c}\text { Testing } \\
\text { accuracy(\%) }\end{array}$ & $\begin{array}{c}\text { Accuracy } \\
\text { standard } \\
\text { deviation(\%) }\end{array}$ & $\begin{array}{c}\text { Testing } \\
\text { time(s) }\end{array}$ & $\begin{array}{c}\text { Time } \\
\text { standard } \\
\text { deviation(\%) }\end{array}$ \\
\hline DELM & 90.71 & 1.78 & 0.2109 & 0.0075 \\
CS-DKELM & 99.97 & 0.44 & 0.0607 & 0.0007 \\
\hline \hline
\end{tabular}

Compared with the direct fault diagnosis of the time-domain signal, our method performs feature recognition on the frequency-domain compressed sampling signal of the original signal. Keeping only $20 \%$ of the original sampling points can achieve better effect than the original diagnostic. And the algorithm has gained good generalization. At present, the real-time requirement of most industrial systems is below 100 milliseconds. The proposed method can well meet the real-time requirements of current industrial systems while maintaining ultra-high accuracy. It is worth mentioning that the 
CS-DKELM framework is not only for a certain scenario of fault diagnosis, but a methodological system [30] of fault diagnosis in complex industrial systems under data-driven conditions. For fault classification problems in different scenarios, we only need to adjust the number of hidden layers and the compression rate to make it adapt better.

\section{Effects of Key Factors}

\section{1) The effect of hidden layer numbers}

In the process of training an effective machine learning model, parameter optimization has always been the focus issue for researchers. Considering the effect of hidden layer numbers on the diagnosis accuracy and generalization ability when training the DKELM network, this paper takes simulation experiments to determine the best hidden layer numbers based on the highest fault diagnosis accuracy. Construct DKELM networks of 2, 3, 4, and 5 layers respectively, and analyze the fault data with the CS-DKELM framework. Fig. 6 shows the effect of the hidden layer numbers in the DKELM network on the accuracy of fault diagnosis.

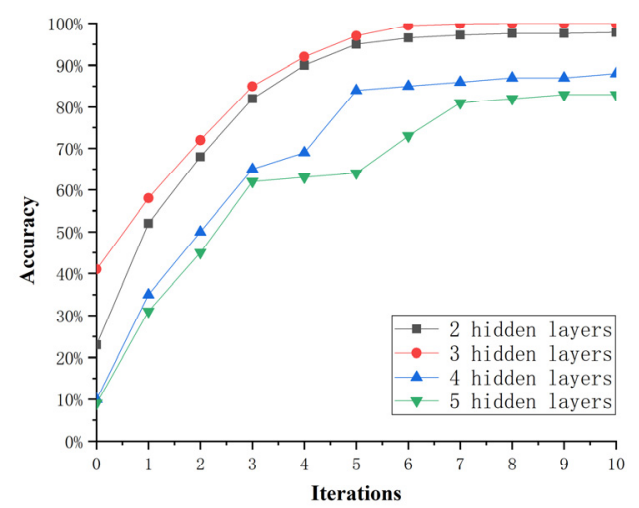

Fig. 6. The effect of the hidden layer numbers.

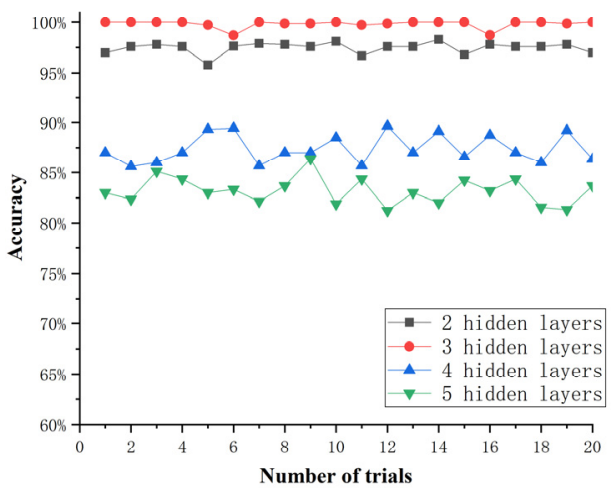

Fig. 7. Accuracy of 20 simulation experiments.

It can be seen that the accuracy of DKELM with 2 hidden layers stabilized at around $97 \%$ after 6 iterations optimization. The accuracy of DKELM with 3 hidden layers can reach more than $99 \%$ after 6 iterations optimization. The accuracy of DKELM with 4 hidden layers stabilized at around $87 \%$ after 5 iterations optimization. The DKELM with 5 hidden layers has the lowest accuracy. The number of PSO iterations is 10, and the experimental results are shown in Fig. 7. The diagnosis accuracy of the DKELM network with 3 hidden layers can reach more than $99 \%$, and the standard deviation does not exceed 0.44 . When the number of hidden layers is greater than 4 , the fault diagnosis accuracy begins to decrease significantly, which proves that the DKELM network with 3 hidden layers has the best performance in this scenario. So, we choose the DKELM network with 3 hidden layers to form the CS-DKELM framework for the research of this paper.

\section{2) The effect of compression ratio}

The size of the measurement matrix is closely related to the compression ratio and the original signal dimensions. Therefore, the compression ratio can be used to control the size of the measurement matrix, thereby controlling the compression degree of the sampled signal. Generally, the higher the compression ratio is, the less sampling points obtained by compressed sensing would be, then the higher the efficiency would be. Because fewer sampling points means smaller sample memory and fewer data processing time. However, there is an upper limit on the compression ratio. Too high a compression rate chosen will cause the random matrix to be unable to obtain the complete information of the original signal, that is, the obtained compressed sampling signal will have serious information loss. This section studies the changes in fault diagnosis accuracy and diagnosis time between $50 \%$ and $95 \%$ of the compression ratio. The result is shown in Fig. 8 .

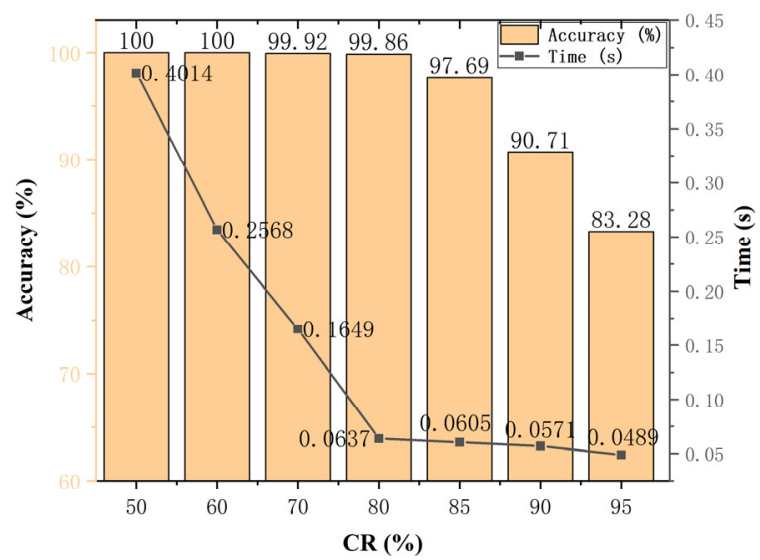

Fig. 8. Diagnosis accuracy and time under different compression ratios.

It can be seen from Fig. 8 that the gradual increase in the compression ratio will reduce the diagnosis time, but the accuracy will also gradually decrease. When the compression ratio reaches $80 \%$, the accuracy ratio decreases insignificantly, and the diagnosis time is greatly reduced. It shows that the accuracy and efficiency of real-time detection can be guaranteed at the same time. Therefore, by weighing the diagnosis accuracy and the diagnosis time, this paper selects a compression ratio of $80 \%$. It also shows that when the sampling frequency is $1 / 5$ of the Nyquist sampling, the compressed sensing method could restore the original signal with high fidelity. The direct use of compressed sampling signals can efficiently implement fault diagnosis while reducing storage costs and diagnosis time. In addition, we found that when the compression ratio reaches $95 \%$, there is still a high diagnostic 
accuracy. Therefore, for some time-sensitive scenarios where diagnosis accuracy required is relatively loose, a high compression ratio can be used, which will greatly reduce the amount of sampling data. Thereby alleviating the pressure of data storage and communication, eventually greatly reducing the time for fault diagnosis.

\section{3) The effect of PSO}

This paper utilizes an $80 \%$ compression ratio and 3 hidden layers to set up comparative experiments. The diagnosis accuracy and diagnosis time is compared between CS-DKELM and the optimization framework CS-DKELM(PSO). The number of hidden layer nodes of the CS-DKELM framework is manually set to $[100,50,100]$, and the optimization interval of the number of hidden layer nodes of CS-DKELM(PSO) is set to [10:1000]. The results of diagnostic accuracy and diagnosis time are shown in Table III.

TABLE III

THE EFFECT OF PSO ON DIAGNOSIS ACCURACY AND TIME

\begin{tabular}{ccccc}
\hline \hline Method & $\begin{array}{c}\text { Testing } \\
\text { accuracy(\%) }\end{array}$ & $\begin{array}{c}\text { Accuracy } \\
\text { standard } \\
\text { deviation(\%) }\end{array}$ & $\begin{array}{c}\text { Testing } \\
\text { time(s) }\end{array}$ & $\begin{array}{c}\text { Time standard } \\
\text { deviation(\%) }\end{array}$ \\
\hline $\begin{array}{c}\text { CS-DKELM } \\
\text { (PSO) }\end{array}$ & 97.31 & 0.72 & 0.0598 & 0.0023 \\
CS-DKELM & 99.97 & 0.44 & 0.0607 & 0.0007 \\
\hline \hline
\end{tabular}

According to the analysis of Table III, the diagnostic accuracy of the CS-DKELM(PSO) is higher than that of the CS-DKELM, but the diagnosis time is slightly increased. Through the analysis of the PSO algorithm, it is known that the diagnosis time is mainly affected by the number of particle swarm iterations. By drawing the fitness curve, we find that the number of particle swarm iterations for the optimal solution is 2 times. In this paper, the initial number of iterations of the particle swarm is set to 10 , so some additional iteration times are added. By setting the maximum number of iterations to jump out of the loop, the time added by the optimization algorithm can be almost ignored. The parameters of the CS-DKELM framework optimized by PSO are shown in Table IV.

TABLE IV

COMPARISON OF FRAMEWORK PARAMETER

\begin{tabular}{ccccc}
\hline \hline Method & $\begin{array}{c}\text { Nodes of } \\
\text { hidden } \\
\text { layers }\end{array}$ & $\begin{array}{c}\text { Regularization } \\
\text { coefficient }\end{array}$ & $\begin{array}{c}\text { Penalty } \\
\text { coefficient }\end{array}$ & $\begin{array}{c}\text { Kernel } \\
\text { parameters }\end{array}$ \\
\hline $\begin{array}{c}\text { CS-DKELM } \\
\text { (PSO) }\end{array}$ & $100-50-100$ & 100 & 100 & 100 \\
CS-DKELM & $65-57-94$ & 111.9482 & 583.2509 & 210.2382 \\
\hline
\end{tabular}

\section{E. Comparison With Other Methods}

To verify the effectiveness of the method in this paper, two classic methods (SVM [31] and DBN [32]) and three latest methods (SPBO-SDAE [33], PSO-DNN [34] and CS-IMSNs [16]) are selected for comparison. The brief settings of these fault diagnosis methods are listed in Table $\mathrm{V}$.

The four evaluation indicators of input data dimension, diagnosis time (training time + test time), diagnosis accuracy, and accuracy standard deviation of the above six methods are respectively compared. The experimental results of the above various methods are shown in Table VI

TABLE V

PARAMETER SETTINGS OF DIFFERENT FAULT DIAGNOSIS METHODS Types Setting

SVM Kernel: Gaussian radial basis function, Box constraint: $1, \gamma: 1 / 1024$

DBN Epoch: 20, Batch size: 64, Learning rate: 0.0002, Optimizer: Adam, Momentum: 0.5 Hidden layers: 46-98-32, Sparse coefficients: SPBO-SDAE $\quad 0.2497-0.3216-0.1415$, Input data zero ratio: $0.05, \mathrm{~N}$ 30, Maximum iterations: 500

PSO-DNN Hidden layers: 600-200-100, Epoch: 50, Learning rate: 0.05 , Optimizer: tanh, Momentum: 0.05 CR: 0.25, Measurement matrices: Gaussian, Epoch: 10, Batch size: 64, Learning rate: 0.0002, Decay: 0.9, Optimizer: RMSProp, Optimizer: ReLU

CS-IMSNs

TABLE VI FRAME PARAMETER COMPARISON

\begin{tabular}{ccccc}
\hline \hline Method & $\begin{array}{c}\text { Input data } \\
\text { dimension }\end{array}$ & $\begin{array}{c}\text { Average } \\
\text { time(s) }\end{array}$ & $\begin{array}{c}\text { Average } \\
\text { accuracy(\%) }\end{array}$ & $\begin{array}{c}\text { Accuracy } \\
\text { standard } \\
\text { deviation(\%) }\end{array}$ \\
\hline $\begin{array}{c}\text { (PSO) } \\
\text { CS-DKELM }\end{array}$ & $1000 \times 960$ & 0.1640 & 99.97 & 0.44 \\
SVM & $1000 \times 4800$ & 10.47 & 90.66 & 1.52 \\
DBN & $1000 \times 2400$ & 13.69 & 96.34 & 3.85 \\
SPBO-SDAE & $1000 \times 4800$ & 29.31 & 98.24 & 2.36 \\
PSO-DNN & $1000 \times 4800$ & 147.22 & 99.61 & 0.87 \\
CS-IMSNs & $1000 \times 1200$ & 270.47 & 99.40 & 1.40 \\
\hline \hline
\end{tabular}

It can be seen from Table VI that due to the compressed sensing method used in the framework of this paper, the input data dimension is much smaller than that of other methods, which will greatly reduce the fault diagnosis time. Compared with the two classic algorithms SVM and DBN, our method has greatly improved both the fault diagnosis time and the diagnosis accuracy. The improved algorithm SPBO-SDAE has a certain similarity with our method, but we have higher accuracy and stronger stability. The optimization algorithm PSO-DNN uses a deep neural network. Although the accuracy has been improved, its diagnosis time is obviously insufficient (the training time is too long) compared with our method. At the same time, the most obvious advantage of our method is that the amount of sample data needed is small, which is very important for reducing storage costs and diagnosis time. Finally, for the CS-IMSNs algorithm, which also uses compressed sensing, due to the need to train deep neural networks of multiple scales, there is a significant gap in training time compared with the method proposed in this paper. Moreover, the fault diagnosis accuracy of our method can reach almost $100 \%$ and can maintain a high degree of stability.

\section{F. Validation on the Actual Hardware Platform}

The PT006 series rotating machinery vibration analysis and fault diagnosis experimental platform XZZZ-1 made by VALENIAN company is used as the verification platform. The platform is composed of a data acquisition system, a variable speed drive motor, a frequency conversion controller, a gearbox, a magnetic powder brake, a tension controller, and a control cabinet. As shown in Fig. 9. 


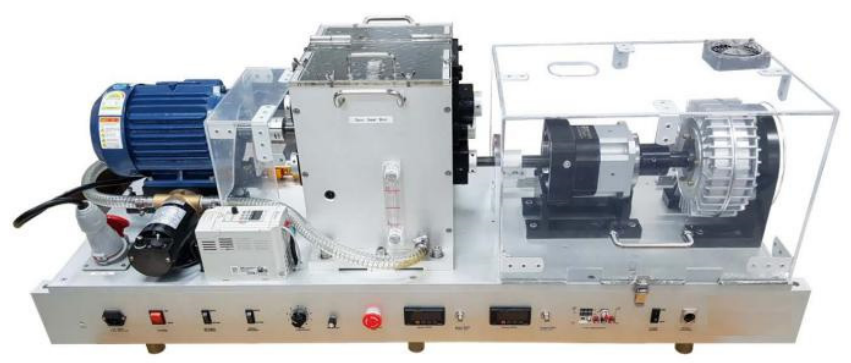

Fig. 9. Rotating machinery vibration analysis and fault diagnosis experimental platform XZZZ-1

In each experiment, the speed of the variable speed drive motor is set to $1450 \mathrm{r} / \mathrm{min}$, and the sampling frequency is $48 \mathrm{kHz}$. To better simulate the actual fault situation, we obtained 2 sets of bearing inner race faults, 2 sets of bearing outer race faults, and 2 sets of ball faults by replacing the prefabricated defective bearings. Besides, 2 sets of gear faults were obtained by replacing the prefabricated defective gears, and 1 set of shaft imbalance fault obtained by adjusting the balance weight of the rotating disc on the shaft. Some faults such as fatigue, plastic deformation, and wear are real natural faults. Other faults such as electrical discharge machining (EDM), electric engraver, and broken teeth are man-made faults. Including the normal state, a total of 10 different health conditions were prefabricated on the above-mentioned platform, as shown in Table VII. The data preprocessing method is the same as this paper. The sliding window intercepts non-overlapping vibration signals. Each sample contains 4800 sampling points, 200 samples are intercepted for each health condition. $70 \%$ of the samples are randomly selected as the training set, while the remaining $30 \%$ as the test set.

TABLE VII

HEALTH STATUS DATA ON THE EXPERIMENTAL PLATFORM

\begin{tabular}{ccll}
\hline \hline Dimension & Label & \multicolumn{1}{c}{ Health condition } \\
\hline & 1 & Bearing inner race fault (Fatigue: pitting) \\
& 2 & Bearing inner race fault (Electric engraver) \\
& 3 & Bearing outer race fault (Fatigue: pitting) \\
4800 & 5 & Bearing outer race fault (Plastic deform: indentations) \\
& 6 & Bearing ball fault (EDM) & \\
& 7 & Gearing ball fault (Electric engraver) & \\
& 8 & Gear tooth surface wear & \\
& 9 & Shaft misalignment & \\
PERFORMANCE OF CS-DKELM ON THE ACTUAL HARDWARE PLATFORM
\end{tabular}

We use the CS-DKELM framework to perform fault diagnosis on the sampled data, and the average value of 20 trials is shown in Table VIII as the result. It can be seen that the average classification accuracy can reach more than $99 \%$ with a good stability. The test time still meets the real-time requirements of industrial systems that are below 100 milliseconds. Our method can be directly applied to industrial embedded platforms as an effective algorithm for mechanical equipment health monitoring. So far, the effectiveness of this framework in the fault diagnosis of rotating machinery has been verified.

\section{CONCLUSION}

Aiming at the limitations of excessive data volume and complex calculation of feature extraction in the process of monitoring the health status of key components of complex industrial systems, this paper proposes an integrated framework for data sampling and fault diagnosis based on CS and DKELM. The framework includes a signal compressed sampling module based on CS theory, which can achieve substantial compression of sampled data while retaining fault information. It also contains a DKELM fault diagnosis module based on extreme learning machine and kernel function. Through the multi-layer non-linear learning of the low-dimensional sampled values of the original signal, the adaptive extraction of fault features and the intelligent diagnosis of health status are realized. The data set of rolling bearings is selected as the experimental data of this paper. We verified the recoverability of the compressed sampled signal. The effectiveness of our method and its influencing factors are studied and compared with existing methods. The analysis shows that the intelligent diagnosis framework proposed in this paper has higher real-time performance and diagnosis accuracy, which are superior to the existing methods. Moreover, only a small amount of monitoring data needed to be sampled in our scheme, which greatly reduces the pressure of transmission, storage and calculation in the process of fault diagnosis. Finally, we applied this method to the actual hardware platform and achieved ideal experimental results, which verified the practical application value of the method proposed in this paper.

\section{REFERENCES}

[1] Xu, Li Da, Eric L. Xu, and Ling Li. "Industry 4.0: state of the art and future trends." International Journal of Production Research, vol. 56, no. 8, pp. 2941-2962, 2018.

[2] Tidriri, Khaoula, et al. "Bridging data-driven and model-based approaches for process fault diagnosis and health monitoring: A review of researches and future challenges." Annual Reviews in Control, vol. 42, pp. 63-81, 2016.

[3] Lei, Yaguo, et al. "A deep learning-based method for machinery health monitoring with big data." Journal of Mechanical Engineering, vol.51, no.21, pp. 49-56, 2015.

[4] Wen, Long, et al. "A new convolutional neural network-based data-driven fault diagnosis method." IEEE Transactions on Industrial Electronics, vol.65, no.7, pp. 5990-5998, 2017.

[5] Gou, Bin, et al. "An intelligent time-adaptive data-driven method for sensor fault diagnosis in induction motor drive system." IEEE Transactions on Industrial Electronics, vol. 66, pp. 9817-9827, 2018.

[6] Kong, Yun, et al. "Data-driven dictionary design-based sparse classification method for intelligent fault diagnosis of planet bearings." Structural Health Monitoring, 2021.

[7] Landau, H. J. "Sampling, data transmission, and the Nyquist rate." Proceedings of the IEEE, vol. 55, no. 10, pp. 1701-1706, 1967.

[8] De Moura, E. P., et al. "Evaluation of principal component analysis and neural network performance for bearing fault diagnosis from vibration signal processed by RS and DF analyses." Mechanical Systems and Signal Processing, vol. 25, no. 5, pp. 1765-1772, 2011.

[9] Hadi Salih, Idris, and Ganesh Babu Loganathan. "Induction motor fault monitoring and fault classification using deep learning probablistic neural network." Solid State Technology, vol. 63, no. 6, pp. 2196-2213, 2020. 
[10] $\mathrm{Li}$, Bo, et al. "Neural-network-based motor rolling bearing fault diagnosis." IEEE transactions on industrial electronics, vol. 47, no. 5, pp. 1060-1069, 2000.

[11] Gupta, Sudha, et al. "Support-vector-machine-based proactive cascade prediction in smart grid using probabilistic framework." IEEE Transactions on Industrial Electronics, vol. 62, no. 4, pp. 2478-2486, 2014.

[12] Amar, Muhammad, Iqbal Gondal, and Campbell Wilson. "Vibration spectrum imaging: A novel bearing fault classification approach." IEEE transactions on Industrial Electronics, vol. 62, no. 1, pp. 494-502, 2014.

[13] Soualhi, Abdenour, et al. "Prognosis of bearing failures using hidden Markov models and the adaptive neuro-fuzzy inference system." IEEE Transactions on Industrial Electronics vol. 61, no. 6, pp. 2864-2874, 2013.

[14] Donoho, David L. "Compressed sensing." IEEE Transactions on information theory, vol. 52, no. 4, pp. 1289-1306, 2006.

[15] Shao, Haidong, et al. "Rolling bearing fault feature learning using improved convolutional deep belief network with compressed sensing." Mechanical Systems and Signal Processing, vol. 100, pp. 743-765, 2018

[16] $\mathrm{Hu}$, Zhong-Xu, et al. "Data-driven fault diagnosis method based on compressed sensing and improved multiscale network." IEEE Transactions on Industrial Electronics, vol. 67, no. 4, pp. 3216-3225, 2019.

[17] Sun, Jiedi, Changhong Yan, and Jiangtao Wen. "Intelligent bearing fault diagnosis method combining compressed data acquisition and deep learning." IEEE Transactions on Instrumentation and Measurement, vol. 67, no. 1, pp. 185-195, 2017.

[18] Tang, Jiexiong, Chenwei Deng, and Guang-Bin Huang. "Extreme learning machine for multilayer perceptron." IEEE transactions on neural networks and learning systems, vol. 27, no. 4, pp. 809-821, 2015.

[19] Zhou, Chi, et al. "Particle swarm optimization (PSO) algorithm." Application Research of Computers, vol. 12, pp. 7-11, 2003.

[20] Baraniuk, Richard, et al. "A simple proof of the restricted isometry property for random matrices." Constructive Approximation, vol. 28, no. 3, pp. 253-263, 2008.

[21] Candes, Emmanuel J., Justin K. Romberg, and Terence Tao. "Stable signal recovery from incomplete and inaccurate measurements." Communications on Pure and Applied Mathematics: A Journal Issued by the Courant Institute of Mathematical Sciences, vol. 59, no. 8, pp. 1207-1223, 2006.

[22] Huang, Guang-Bin, Qin-Yu Zhu, and Chee-Kheong Siew. "Extreme learning machine: theory and applications." Neurocomputing, vol. 70, no. 1-3, pp. 489-501, 2006.

[23] AlBahar, Areej, Inyoung Kim, and Xiaowei Yue. "A Robust Asymmetric Kernel Function for Bayesian Optimization, With Application to Image Defect Detection in Manufacturing Systems." IEEE Transactions on Automation Science and Engineering, 2021.

[24] James, Gareth, et al. "Support vector machines." An introduction to statistical learning. Springer, New York, NY, pp. 367-402, 2021.

[25] Huang, Guang-Bin. "An insight into extreme learning machines: random neurons, random features and kernels." Cognitive Computation, vol. 6, no. 3, pp. 376-390, 2014.

[26] Minh, Ha Quang, Partha Niyogi, and Yuan Yao. "Mercer's theorem, feature maps, and smoothing." International Conference on Computational Learning Theory. Springer, Berlin, Heidelberg, 2006.

[27] Q. Tang, W. Qiu and Y. Zhou, "Classification of Complex Power Quality Disturbances Using Optimized S-Transform and Kernel SVM," IEEE Transactions on Industrial Electronics, vol. 67, no. 11, pp. 9715-9723, Nov. 2020.

[28] Smith, Wade A., and Robert B. Randall. "Rolling element bearing diagnostics using the Case Western Reserve University data: A benchmark study." Mechanical systems and signal processing, vol. 64, pp. 100-131, 2015.

[29] Mao, Wentao, et al. "Bearing fault diagnosis with auto-encoder extreme learning machine: A comparative study." Proceedings of the Institution of Mechanical Engineers, Part C: Journal of Mechanical Engineering Science, vol. 231, no. 8, pp. 1560-1578, 2017

[30] Liu, Jia, et al. "Boost Precision Agriculture with Unmanned Aerial Vehicle Remote Sensing and Edge Intelligence: A Survey." Remote Sensing, vol. 13, no. 21, pp. 4387, 2021.

[31] Konar, P., and P. Chattopadhyay. "Bearing fault detection of induction motor using wavelet and Support Vector Machines (SVMs)." Applied Soft Computing, vol. 11, no. 6, pp. 4203-4211, 2011.
[32] Shao, Haidong, et al. "Rolling bearing fault diagnosis using an optimization deep belief network." Measurement Science and Technology, vol. 26, no. 11, pp. 115002, 2015.

[33] Du Xianjun, Jia Liangliang. "Rolling bearing fault diagnosis based on the optimized stack noise reduction autoencoder." Journal of Jilin University(Engineering and Technology Edition), pp. 1-10, 2021.

[34] Jia, Feng, et al. "Deep neural networks: A promising tool for fault characteristic mining and intelligent diagnosis of rotating machinery with massive data." Mechanical Systems and Signal Processing, vol. 72, pp. 303-315, 2016.

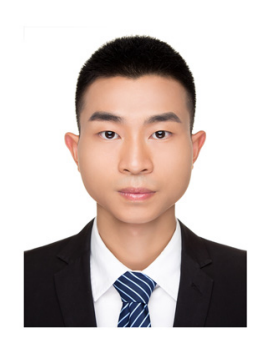

Nanliang Shan received the B.E. degree in communication engineering from Xiamen University, China, in 2018. He is currently working toward the Ph.D. degree in electrical engineering with the National Key Laboratory of Science and Technology on Vessel Integrated Power System, Naval University of Engineering, Wuhan, China.

His current research interests include edge intelligence, lightweight computing, prognostic and health management.

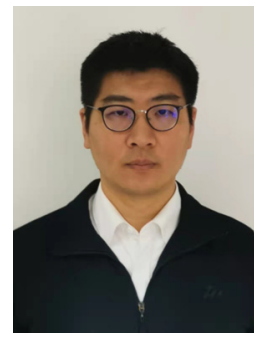

Xinghua $\mathbf{X u}$ received the B.E. and M.E. degrees from National University of Defense Technology, Changsha, China, in 2004 and 2007 and the Ph.D. degree from the National Key Laboratory of Science and Technology on Vessel Integrated Power System, Naval University of Engineering, Wuhan, China, in 2016.

$\mathrm{He}$ is currently an Associate Professor with the National Key Laboratory of Science and Technology on Vessel Integrated Power System, Naval University of Engineering, Wuhan, China. His research interests include cyber physical system, complex industrial control systems, prognostic and health management.

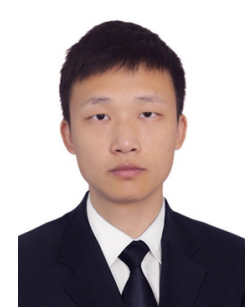

Shaohua Qiu received the B.E. degree from Wuhan University, Wuhan, China, in 2011, and the M.S. and Ph.D. degree from National University of Defense Technology, Changsha, China, in 2013 and 2018, respectively.

$\mathrm{He}$ is currently an Associate Professor with the National Key Laboratory of Science and Technology of Vessel Integrated Power System, Naval University of Engineering, Wuhan, China. His current research interests include deep learning, image analysis, mechanical fault diagnosis.

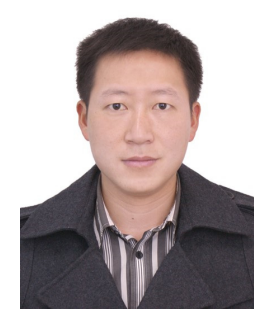

Xianqiang Bao received the B.E. degree from Huazhong University of Science and Technology, Wuhan, China, in 2009, and the M.E. and Ph.D. degrees from National University of Defense Technology, Changsha, China, in 2011 and 2016 $\mathrm{He}$ was a visiting Ph.D. student with Georgia Institute of Technology from 2013 to 2015.

$\mathrm{He}$ is currently an Associate Professor with the National Key Laboratory of Science and Technology on Vessel Integrated Power System, Naval University of Engineering, Wuhan, China. His current research interests include edge intelligence, industrial big-data, prognostic and health management. 